\title{
Editorial
}

\section{Scaling, Uncertainty, and 3D Coupled Code Calculations in Nuclear Technology}

\author{
Cesare Frepoli ${ }^{1}$ and Alessandro Petruzzi ${ }^{2}$ \\ ${ }^{1}$ LOCA Integrated Services I, Westinghouse Electric Company, Pittsburgh, PA 15230-0350, USA \\ ${ }^{2}$ Nuclear Research Group San Piero a Grado (NRGSPG), Department of Mechanical, Nuclear and Production Engineering, \\ University of Pisa, Pisa, 56100, Italy
}

Correspondence should be addressed to Alessandro Petruzzi, a.petruzzi@ing.unipi.it

Received 22 May 2008; Accepted 22 May 2008

Copyright (C) 2008 C. Frepoli and A. Petruzzi. This is an open access article distributed under the Creative Commons Attribution License, which permits unrestricted use, distribution, and reproduction in any medium, provided the original work is properly cited.

System codes such as RELAP, TRACE, CATHARE, or ATHLET are currently used by designer/vendors of NPPs, by utilities, licensing authorities, research organizations including universities, nuclear fuel companies, and by technical supporting organizations. The objectives of using the codes may be quite different, ranging from design or safety assessment to simply understanding the transient behavior of a simple system. However, the application of a selected code must be proven to be adequate to the performed analysis. Thus considerable research efforts have been spent in the last three decades, and as a consequence a wide range of activities has recently been completed in the area of system thermalhydraulics. Problems have been addressed, solutions to which have been at least partly agreed upon on international ground. These include the need for best-estimate system codes, the general code qualification process, the proposal for nodalization qualification as well as attempts aiming at qualitative and quantitative accuracy evaluations. Moreover, complex uncertainty methods have been proposed, following a pioneering study which attempted, among other things, to account for user effects on code results.

Based on the above considerations, this special issue mostly focuses on the development and application of bestestimate codes emphasizing the role of the scaling, best estimate and uncertainty, and $3 D$ coupled code calculations analyses.

In general terms, scaling indicates the need for the process of transferring information from a model to a prototype. In system thermal hydraulics, a scaling process, based upon suitable physical principles, aims at establishing a correlation between phenomena expected in an NPPtransient scenario as (a) phenomena measured in smaller scale facilities, or (b) phenomena predicted by numerical tools qualified against experiments performed in small-scale facilities. In connection with this point, owing to limitations of the equations at the basis of system codes, the scaling issue may constitute an important source of uncertainties in code applications.

By definition, a best-estimate analysis (the term "bestestimate" is usually used as a substitute for "realistic") is an accident analysis which is free of deliberate pessimism regarding selected acceptance criteria, and is characterized by applying best-estimate codes along with nominal plant data and best-estimate initial and boundary conditions. However, notwithstanding the important achievements and progress made in recent years, the predictions of the best-estimate system codes are not exact but remain uncertain because (a) the assessment process depends upon data almost always measured in small-scale facilities and not in the full-power reactors; (b) the models and the solution methods in the codes are approximate. In some cases, fundamental laws of physics are not considered. Consequently, the results of the code calculations may not be applicable to give exact information on the behavior of a nuclear power plant (NPP) during postulated accident scenarios. Therefore, bestestimate predictions of NPP scenarios must be supplemented by proper uncertainty evaluations in order to be meaningful. The term "best-estimate plus uncertainty" was coined for indicating an accident analysis which (1) is free of deliberate pessimism regarding selected acceptance criteria, (2) uses a BE code, and (3) includes uncertainty analysis. Thus, the word "uncertainty" and the need for uncertainty evaluation are strictly connected with the use of BE codes.

Nowadays, advanced $3 D$ coupled neutron-kinetics/ thermal-hydraulics computer tools along with powerful computers can perform realistic best-estimate analyses of 
complex power plant transients. The interaction between thermal-hydraulics and neutron kinetics is relevant for both the safety and the design of existing nuclear reactors. The results from the application of coupled computational tools provide new insights into the conservatisms for the specification of relevant operational safety margins and can imply new optimizations of emergency operating procedures in existing plants. They also improve knowledge of the physical phenomena in nuclear water reactor technology and can specifically shed light on the interaction between thermal-hydraulics and neutron kinetics that still can challenge the design and the operation of nuclear power plants.

This special issue collects selected lectures delivered at the 3D S.UN.COP (Scaling, Uncertainty, and 3D COuPled code calculations) seminars-trainings whose aim is to transfer competence, knowledge, and experience from about 30 recognized international experts coming from more than 10 different countries and institutions to analysts with a suitable background in nuclear technology. The program of the 3D S.UN.COP offers each year about 60 presentations and 100 hours of parallel code hands-on training subdivided in three weeks and covering the following topics: (a) system codes: evaluation, application, modeling and scaling; (b) international standard problems; (c) best-estimate in system code applications and uncertainty evaluation; (d) qualification procedures; (e) methods for sensitivity and uncertainty analysis; (f) relevant topics in best-estimate licensing approach; (g) industrial applications of the best-estimateplus-uncertainty methodology; (h) coupling methodologies and applications; (i) computational fluid dynamics codes. From the other side, the parallel hands-on training sessions on numerical codes (such as CATHARE, CATHENA, RELAP5, TRACE, and PARCS) allow the participants to achieve the capability to set up, run, and evaluate the results of a numerical tool through the application of the proposed qualitative and quantitative accuracy evaluation procedures. Finally, the 3D S.UN.COP seminars provides a forum for exchanges of ideas through scientific presentations and dialogue among representatives of the worlds of academy, research laboratories, industry, regulatory authorities, and international institutions.

In the first paper, A. Petruzzi et al. emphasized the role of the computer code user that represents one of the main sources of uncertainty influencing the results of system code calculations. This influence is commonly known as the "user effect" and stems from the limitations embedded in the codes as well as from the limited capability of the analysts to use the codes. The paper describes a systematic approach to training code users who, upon completion of the training, should be able to perform calculations making the best possible use of the capabilities of best-estimate codes.

In the second paper, A. Petruzzi, and F. D'Auria presented the commonly used system thermal-hydraulic codes such as RELAP, TRACE, CATHARE, or ATHLET for reactor-transient simulations. Whereas the first system codes, developed at the beginning of the 1970s, utilized the homogenous equilibrium model with three balance equations to describe the two-phase flow, nowadays the more advanced system codes are based on the so-called "two-fluid model" with separation of the water and vapour phases, resulting in systems with at least six balance equations. However, notwithstanding the huge amounts of financial and human resources invested, the results predicted by the code are still affected by errors whose origins can be attributed to several reasons as model deficiencies, approximations in the numerical solution, nodalization effects, and imperfect knowledge of boundary and initial conditions. In this context, the existence of qualified procedures for a consistent application of qualified thermal-hydraulic system code is necessary and implies the drawing up of specific criteria through which the code-user, the nodalization, and finally the transient results are qualified.

In "International Standard Problems and Small Break Loss-Of-Coolant Accident (SBLOCA)," N. Aksan considered five small break LOCA-related ISPs since these were used for the assessment of the advanced best-estimate codes. The considered ISPs deal with the phenomenon typical of small break LOCAs in Western design PWRs. The experiments in four integral test facilities, LOBI, SPES, BETHSY, ROSA IV/LSTF, and in the recorded data during a steam generator tube rupture transient in the DOEL-2 PWR (Belgium) were the basis of ISP calculations. The statistical evaluation of the general data obtained from these ISPs is summarized. Some lessons learned from these small break LOCA ISPs are identified in relation to code deficiencies and capabilities, progress in the code capabilities, possibility of scaling, and various additional aspects. ISPs are providing unique material and benefits for some safety related issues. Some of the technical findings and benefits provided by small break LOCA ISPs are provided as conclusions and recommendations.

In the next paper, A. Petruzzi, and F. D'Auria presented the evaluation of uncertainty methodologies as necessary supplement of best-estimate calculations performed to understand accident scenarios in water-cooled nuclear reactors. The needs come from the imperfection of computational tools, on the one side, and the interest in using such a tool to get more precise evaluation of safety margins. The paper reviews the salient features of two independent approaches for estimating uncertainties associated with predictions of complex system codes. Namely, the propagations of code input error and calculation output error constitute the keywords for identifying the methods of current interest for industrial applications. Throughout the developed methods, uncertainty bands can be derived (both upper and lower) for any desired quantity of the transient of interest. For one case, the uncertainty method is coupled with the thermal-hydraulic code to get the code with capability of internal assessment of uncertainty, whose features are discussed in more detail.

The task of regulatory body staff reviewing and assessing a realistic large break loss-of-coolant accident evaluation model is discussed by R. Galetti in the next paper facing the actual regulatory licensing environment related to the acceptance of the analysis of emergency core cooling system performance. Especially, focus is directed to the question of how to fulfill the requirement of quantifying the uncertainty in the calculated results when they are compared to the 
acceptance criteria for this system. When using a realistic evaluation model to analyze the loss-of-coolant accident, different approaches have been used in the licensing arena. The Brazilian regulatory body has concluded that, in the current environment, the independent regulatory calculation is recognized as a relevant support for the staff decision within the licensing framework of a realistic analysis.

In the sixth paper, $H$. Glaeser summarized the basic techniques of the GRS uncertainty method together with applications to a large break loss-of-coolant accident on a reference reactor as well as on an experiment simulating containment behavior. A significant advantage of this methodology is that no a priori reduction in the number of uncertain input parameters by expert judgement or screening calculations is necessary to limit the calculation effort. All potentially important parameters may be included and the number of calculations needed is independent of the number of uncertain parameters accounted for in the analysis. A challenge in performing uncertainty analyses with the GRS methodology is the specification of ranges and probability distributions of input parameters.

C. Frepoli presented the paper entitled "An Overview of Westinghouse Realistic Large Break LOCA Evaluation Model." Since the 1988 amendment of the 10 CFR 50.46 rule in 1988, Westinghouse has been developing and applying realistic or best-estimate methods to perform LOCA safety analyses. Westinghouse methodology is based on the use of the WCOBRA/TRAC thermal-hydraulic code. The paper starts with an overview of the regulations and its interpretation in the context of realistic analysis. The CSAU (code scaling, applicability, and uncertainty) roadmap is reviewed in the context of its implementation in the Westinghouse evaluation model. An overview of the code (WCOBRA/TRAC) and methodology is provided. Finally, the recent evolution to nonparametric statistics in the current edition of the Westinghouse methodology is discussed. Sample results of a typical large break LOCA analysis for a PWR are provided.

The next paper by R. Martin and L. O'Dell illustrates the development considerations of AREVA NP Inc.'s realistic LBLOCA analysis methodology. The AREVA NP RLBLOCA methodology is a CSAU-based methodology for performing best-estimate large-break LOCA analysis. The methodology addresses all of the expressed steps of the CSAU process. The key challenge to this process has been the defense of declared engineering judgment and the demonstration of the methodologies' range of applicability. This was accomplished by careful characterization of dominant LOCA parameters and emphasis on validation through sensitivity studies and the statistical nature of the methodology. The generic AREVA NP RLBLOCA methodology was approved by the USNRC in April 2003 and is now being applied to several nuclear power plants serviced by AREVA NP Inc.

In the next paper, D. Novog and P. Sermer provided a novel and robust methodology for determination of nuclear reactor trip set points, which accounts for uncertainties in input parameters and models, and for the variations in operating states that periodically occur. The paper presents the general concept used to determine the actuation set points considering the uncertainties and changes in initial conditions, and allowing for safety system instrumentation redundancy. The results demonstrate unique statistical behavior with respect to both fuel and instrumentation uncertainties, which has not previously been investigated.

F. Reventos et al. illustrated the usefulness of computational analysis for operational support in the paper before the last. In the first part, he described the specific aspects of thermal-hydraulic analysis tasks related to operation and control and, in the second part, they briefly presented the results of three examples of performed analyses. All the presented examples are related to actual situations in which the scenarios were studied by analysts using thermalhydraulic codes and prepared nodalizations. The paper also includes a qualitative evaluation of the benefits obtained through thermal-hydraulic analyses aiming at supporting operation and plant control.

In the last paper, $H$. Ikeda et al. reviewed activities relevant to the boiling water reactor (BWR) stability phenomenon, which has a coupled neutronic and thermalhydraulic nature, from the viewpoint of model and code developments. Industrial organizations have developed and improved the BWR stability analysis using computational tools specific for the reduced-order frequency-domain and three-dimensional time-domain codes. The first category is currently applied to the BWR stability design analysis, while the latter has been exploited to understand the complicated phenomena related to BWR stability. Proposals to apply bestestimate analysis code with the statistical safety evaluation methodology are currently under study. This will allow better evaluation of the stability exclusion region, and will be consequently applied to the BWR plants with the extended core power uprate.

We believe that the collection of papers in this special issue illustrates the great variety of topics and problems in the nuclear technology for which advanced tools are available and applicable.

Finally, we would like to take the opportunity to express our thanks to all authors who have submitted papers to this special issue and to our colleagues who devoted their valuable time reviewing these manuscripts.

Cesare Frepoli Alessandro Petruzzi 

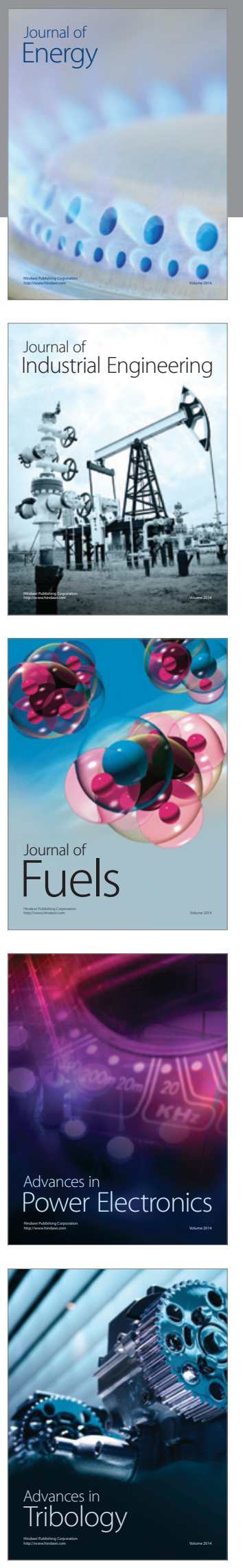
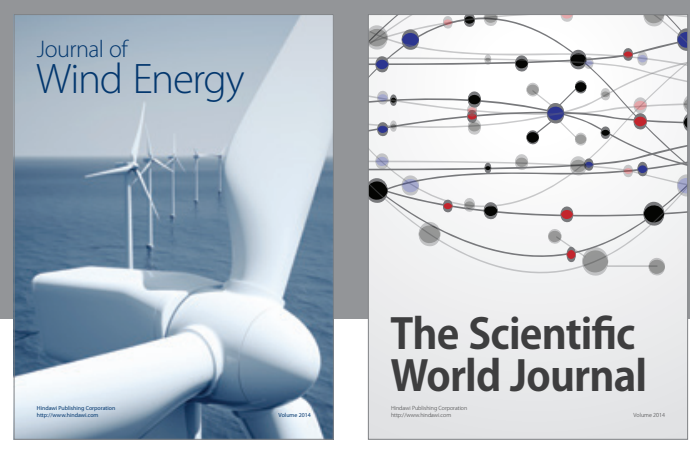

The Scientific World Journal

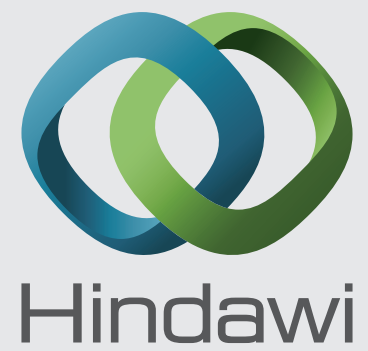

Submit your manuscripts at http://www.hindawi.com
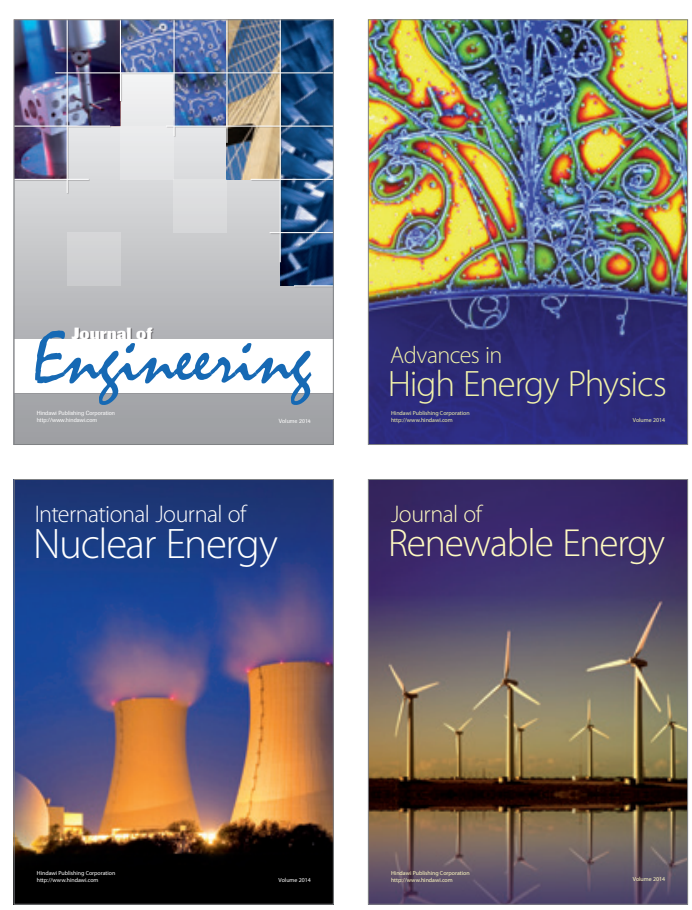

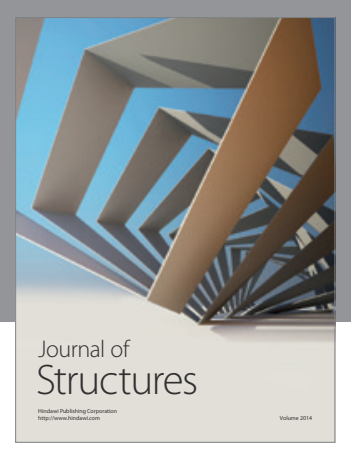

Rotating
Mechinery
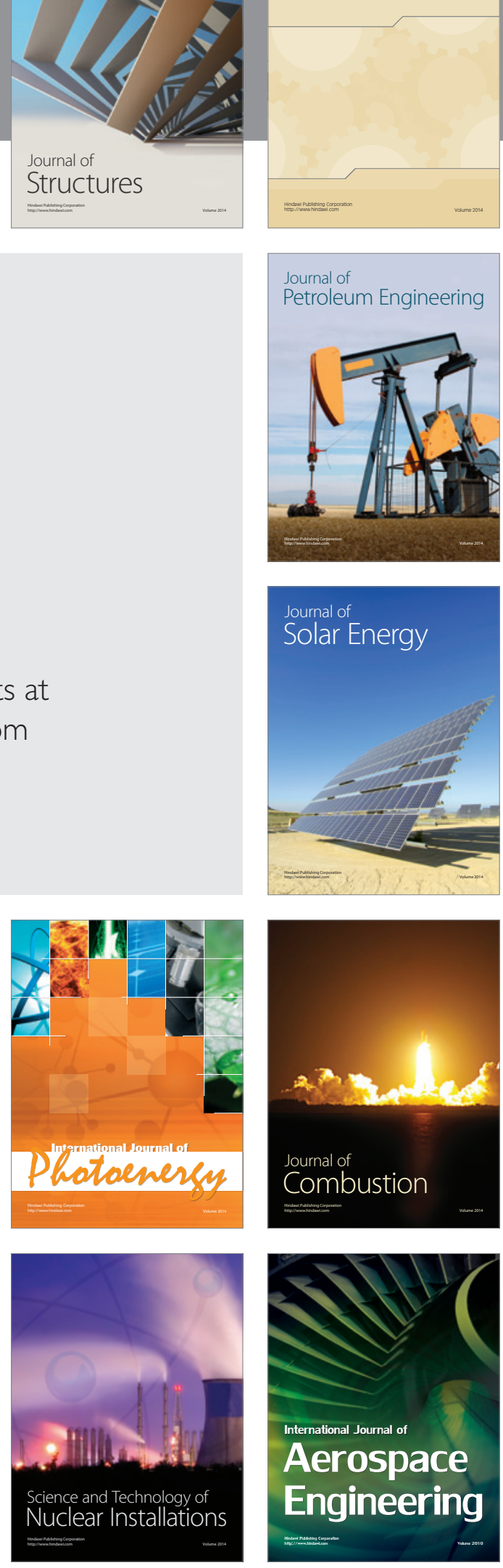